\title{
The Use of Ultrasonography, Transient Elastography, APRI and FIB-4 to Measure Liver Steatosis and Fibrosis in HIV-Positive Patients Not Co- Infected with Hepatitis Viruses with Hypertransaminasemia of Unknown Etiology on HAART
}

\author{
Ilaria Izzo, Luciano Biasi, Monia Mendeni, Katiela Prestini, Andrea Vavassori, Emanuele Focà, \\ Eugenia Quiros-Roldan, Giampiero Carosi and Carlo Torti*
}

Institute of Infectious and Tropical Disease, University of Brescia, Italy

\begin{abstract}
Background: HIV positive patients may be affected by hypertransaminasemia notwithstanding they are not coinfected with HCV and HBV.

Aims: To understand the causes of this abnormality and what correlates are in terms of ultrasonic transient elastography (UTE) and ultrasonography (U) features and fibrosis scores.

Methods: HIV positive patients with hypertransaminasemia have been studied. They underwent UTE and U. Non-invasive fibrosis scores (APRI and FIB-4) were calculated. Moreover, they underwent immunological and virological tests to exclude known causes of liver damage (including alcohol abuse).

Results: Among 24 patients, 3 presented a progressive fibrosis at UTE. 3/3 with progressive fibrosis and further 14 patients among the entire sample had steatosis at U. Using non-invasive fibrosis scores, no patients had significant fibrosis, while 5 patients had mild fibrosis. 14 patients had hepatomegaly independently from steatosis. One patient has progressive fibrosis at UTE and mild fibrosis at both APRI and FIB-4, while 2 patients had fibrosis only at UTE, 2 only at APRI and 1 at both APRI and FIB-4, but not at UTE. Alcoholaemia was negative in all patients, confirming anamnestic information. No other causes of liver disease were found.

Conclusions: In this series, more than $50 \%$ of patients had steatosis at U. Discordance between the non-invasive methods to estimate liver fibrosis were found. Further prospective studies are necessary to assess concordance between these methods and liver biopsy and assess the prognostic value of UTE, APRI and FIB-4 for liver complications in HIV monoinfected patients so as to improve diagnostic algorithms.
\end{abstract}

Keywords: HIV infection, hypertransaminasemia, steatosis, fibrosis.

\section{INTRODUCTION}

Hypertransaminasemia may present in HIV-positive patients on highly active antiretroviral therapy (HAART), even though they are not co-infected with main hepatotropic viruses (HBV and $\mathrm{HCV}$ ). The etiology of liver injury is a complex phenomenon which involves direct drug toxicity, but also an idiosyncratic response, mitochondrial toxicity and immune reconstitution have been implicated [1].

Several studies have found that hepatic steatosis is highly prevalent in HIV-positive patients, in particular in those coinfected with $\mathrm{HCV}$ and in those treated with nucleoside reverse transcriptase inhibitors (NRTI), a drug-class implicated in mitochondrial toxicity; in its turn, liver steatosis can progress to fibrosis $[2,3]$.

Ultrasonography is a standard method to diagnose hepatic steatosis, while the gold standard for the diagnosis of hepatic fibrosis is liver biopsy, but its invasive nature [4], and potential sampling errors [5] support the use of

\footnotetext{
*Address correspondence to this author at the Institute of Infectious and Tropical Diseases, University of Brescia, P.le Spedali Civili, 1, 25123 Brescia, Italy; Fax: +39.030.303061; E-mail: torti@med.unibs.it
}

ultrasonic transient elastography (FibroScan ${ }^{\mathrm{TM}}$ ) as a measure of hepatic fibrosis. Yoneda et al. [6] demonstrated a significant positive correlation between liver stiffness (the parameter indicating fibrosis at ultrasonic transient elastography) and the severity of liver fibrosis in HIVnegative patients with non-alcoholic fatty liver disease (NAFLD), suggesting the utility of elastometry in routine clinical practice.

Moreover, direct and indirect markers of fibrosis may be used alone or in combination. The utility of these markers has been evaluated mostly in HCV mono-infected or HIV/HCV co-infected patients (i.e. APRI, FibroTest). Other tests (e.g. FIB-4, FORNS) have been specifically designed for HIV/HCV co-infection in particular. Cozzi-Lepri et al. demonstrated, in a mixed population of HIV-mono-infected and HCV/HBV-co-infected patients, that FIB-4 is a strong predictor of severe liver-related morbidity and mortality [7]. Moreover, in two recent cross-sectional studies performed in HIV-mono-infected patients, APRI score and FIB4 score were used to estimate the prevalence of severe liver fibrosis and its risk factors $[8,9]$.

We have conducted an explorative survey in a month period of our patients with hypertransaminasemia 
nothwithstanding negative $\mathrm{HBV} / \mathrm{HCV}$ antibody tests to assess concordance between steatosis (measured by standard ultrasonography) and hepatic fibrosis (measured by FibroScan $^{\mathrm{TM}}$ and non-invasive biochemical scores).

\section{MATERIALS AND METHODS}

In October 2007, HIV positive patients with hypertransaminasemia whose cause was indetermined (defined as a single determination of $\mathrm{ALT}>50 \mathrm{IU} / \mathrm{L}$ ) were selected. Patients with known risk factors for liver damage (alchool abuse, HBV/HCV co-infections as determined by standard antibody tests, use of hepatotoxic drugs except from antiretroviral drugs) were excluded.

All selected patients were asked to undergo ultrasonography, ultrasonic transient elastography and blood tests to identify possible underlying causes of the liver damage (including HCV-RNA and HBV-DNA to exclude active viral hepatites notwithstanding $\mathrm{HBV} / \mathrm{HCV}$ antibody negativity, auto-antibodies to exclude autoimmune liver disease and dosage of serum alcohol to confirm anamnestic absence of alcohol abuse).

Transient elastography was performed with Fibroscan ${ }^{\mathrm{TM}}$ (EchoSens, Paris, France). The APRI score was calculated as [AST/(upper limit normal)/platelet count $\left.\left(10^{\wedge} 9 / \mathrm{L}\right)\right]$ [10]. The FIB4 was calculated as AST(UI/L) x age(years)/platelet count $\left(10^{\wedge} 9 / \mathrm{L}\right) \times$ x $[$ ALT (UI/L)]1/2 [11]. The cutoffs used were chosen by previous recommended cutoffs for the APRI for the separation of mild and significant fibrosis in HCV mono-infected and $\mathrm{HIV} / \mathrm{HCV}$ co-infected patients [11]. If the APRI is $>1.5$, patients can be classified as having significant fibrosis [fibrosis stage $(F) \geq 2$, with a positive predictive value ranging from 66 to $100 \%$ ]. For the FIB4 the cutoff was chosen according to validation studies in $\mathrm{HCV}$ monoinfected and HIV/HCV coinfected patients (i.e.>3.25) [12-14].

The blood exams included ANA, AMA, SMA, LKM, CMV-DNA, HBsAg, HBcAb, HBV-DNA, HCV-RNA and alcoholaemia.

\section{RESULTS}

Twenty-four HIV positive patients were studied; their characteristics and antiretroviral treatment received are summarized in Table $\mathbf{1}$. They were all virologically suppressed (HIV RNA $<50$ copies/ml for at least 6 months) and their CD4+ T-cell counts were $\geq 200$ cells $/ \mathrm{mm}^{3}$ (mean 550 cells $/ \mathrm{mm}^{3}$, standard deviation (SD) 216.7 cells $/ \mathrm{mm}^{3}$ ).

At ultrasonic transient elastography 21/24 (87.5\%) patients presented a stiffness $<7 \mathrm{KPa}$ (commonly defined as "mild fibrosis") and 3/24 (12.5\%) a stiffness between 7.1 and $9 \mathrm{KPa}$ (defined as "progressive fibrosis") (see patients \# 6, 7 and 13).

No significant fibrosis was detected with APRI $(>1.5)$ and FIB-4 (>3.25), while 5/24 patients presented mild fibrosis with these non-invasive markers (0.6 to 1.5 for APRI and 1.45 to 3.25 for FIB-4). Only one patient (\#13) presented progressive fibrosis at elastography and mild fibrosis at APRI and FIB-4, while 2 patients had fibrosis only at elastography (\# 6 and 7), 2 only at APRI (\#17 and 19), and 1 at both APRI and FIB-4, but not at elastography (\#24).
At standard ultrasonography, liver steatosis was found in $14 / 24(58.3 \%)$ patients (11 among 21 with mild fibrosis and all the 3 patients with progressive fibrosis at elastometry; 3 with mild fibrosis at APRI and 1 with mild fibrosis at both APRI and FIB-4). Maximal diameter of the portal vein was $13 \mathrm{~mm}$ and this diameter did not correlate with stiffness measures. Ten of 14 patients with steatosis had hepatomegaly at ultrasonography; 3 patients had hepatomegaly without evidence of liver steatosis. 9/14 patients with steatosis presented also dyslipidemia at blood exams (defined as total cholesterol $>200 \mathrm{mg} / \mathrm{dL}$ and/or TG > $150 \mathrm{mg} / \mathrm{dL}) .4$ patients with dyslipidemia did not show steatosis at standard ultrasonography. Only $1 / 24$ patients had diabetes and he presented steatosis at standard ultrasonography and mild fibrosis at elastometry.

Alcoholaemia was negative in all patients, confirming the anamnestic information. AMA and LKM were negative in all patients, while ANA were positive in 5 and SMA in further 2 patients. CMV-DNA, HCV-RNA and HBV-DNA were negative in all patients. Body mass index was $>25 \mathrm{Kg} / \mathrm{m}^{2}$ in $14 / 24$ patients $(10 / 14$ with liver steatosis and $3 / 3$ with progressive fibrosis at UTE). Amongst 6 patients with isolated $\mathrm{HBcAb}, 2$ had progressive fibrosis at ultrasonic transient elastography examination.

\section{DISCUSSION}

In the present work, we studied a limited, but well characterized, group of HIV positive patients with hypertransaminasemia in absence of $\mathrm{HBV} / \mathrm{HCV}$ coinfections; several possible causes of liver damage have been considered in the diagnostic work-up, and standard ultrasonography and transient elastography examinations were performed to assess liver steatosis and fibrosis. Noninvasive biochemical markers of fibrosis (APRI and FIB-4) were also evaluated.

Our study showed moderate concordance between elastometry, APRI and FIB-4. A recent study conducted among HIV-HCV co-infected patients showed that some non-invasive indexes of fibrosis (APRI, FORNS, FibroTest, Shasta) had acceptable performance in the diagnosis of advanced fibrosis and cirrhosis but are still not adequate for diagnosing early stages of fibrosis [12]. Gonzàlez-Guilabert and collaborators assessed that, in $\mathrm{HCV}$-infected patients, elastography, APRI and FIB-4 were acceptable in predicting the presence or absence of mild fibrosis and the absence of advanced fibrosis [15]. However, the low or moderate agreement with liver biopsy and low sensitivities in the detection of mild fibrosis indicated that liver biopsy continues to be required when the results of other methods are discordant or indeterminate [15]. Our study conducted in HIV mono-infected patients highlighted the discordance between the non invasive methods, clearly indicating the importance of concordance studies between these methods and liver biopsy.

Non-alcoholic fatty liver disease (NAFLD), that occurs in absence of chronic infection with viral hepatites or a history of alcohol abuse, is the most common cause of elevated serum transaminases in Western countries in the general population [16]. Few studies have been conducted on NAFLD in HIV-positive patients, suggesting that it may 
Table 1. Characteristics of the Study Patients

\begin{tabular}{|c|c|c|c|c|c|c|c|c|c|c|c|c|c|c|c|c|c|}
\hline 1 & 24 & $F$ & 236 & $\mathrm{~N}$ & 2004 & NVP+ FTC/TDF & $\mathrm{EFV}, 3 \mathrm{TC}$ & 78 & 20.6 & $\mathrm{~N}$ & $\mathrm{~N}$ & 328 & $<50$ & $\mathrm{~N}$ & 5.8 & 0.5 & 0.59 \\
\hline 5 & 60 & M & 359 & $\mathrm{~N}$ & 2002 & $\begin{array}{l}\text { ATV/r+ } \\
\text { 3TC/ABC }\end{array}$ & AZT, LPV/r, FTC, TDF & 81 & 28.9 & $\mathrm{~N}$ & $\mathrm{~N}$ & 798 & $<50$ & $\mathrm{Y}$ & 3.6 & 0.5 & 1.31 \\
\hline 6 & 44 & M & 86 & $\mathrm{~N}$ & 2000 & $\mathrm{FSV} / \mathrm{r}+\mathrm{AZT} / 3 \mathrm{TC}$ & ddI, d4T, NVP, ABC, TDF & 57 & 26.4 & $\mathrm{Y}$ & $\mathrm{N}$ & 434 & $<50$ & $\mathrm{Y}$ & 7.7 & 0.3 & 0.74 \\
\hline 7 & 63 & M & 402 & $\mathrm{~N}$ & 1998 & $\begin{array}{l}\text { ATV/r+ } \\
\text { 3TC/ABC }\end{array}$ & AZT, ddI & 52 & 27.5 & Y & $\mathrm{N}$ & 439 & $<50$ & $\mathrm{Y}$ & 8.8 & 0.4 & 1.35 \\
\hline 8 & 32 & M & 288 & $\mathrm{~N}$ & 2007 & $\mathrm{LPV} / \mathrm{r}+\mathrm{FTC} / \mathrm{TDF}$ & ATV/r & 57 & 21.6 & $\mathrm{Y}$ & $\mathrm{N}$ & 590 & $<50$ & $\mathrm{~N}$ & 3.4 & 0.1 & 0.30 \\
\hline 9 & 50 & $\mathrm{~F}$ & 189 & $\mathrm{~N}$ & 1997 & NVP+ FTC/TDF & AZT, ddI, ddC, 3TC, EFV & 51 & 19.4 & $\mathrm{~N}$ & $\mathrm{~N}$ & 633 & $<50$ & $\mathrm{~N}$ & 3.9 & 0.2 & 0.61 \\
\hline 10 & 41 & M & 171 & $\mathrm{~N}$ & 2004 & NVP+ FTC/TDF & LPV/r, 3TC, AZT, NVP & 64 & 33.9 & Y & $\mathrm{N}$ & 588 & $<50$ & Y & 4.6 & 0.3 & 0.64 \\
\hline 15 & 37 & M & 18 & $\mathrm{~N}$ & 2003 & $\mathrm{ATV} / \mathrm{r}+\mathrm{FTC} / \mathrm{TDF}$ & ddI, EFV, 3TC & 64 & NK & Y & $\mathrm{N}$ & 401 & $<50$ & $\mathrm{~N}$ & 5.4 & 0.4 & 1.13 \\
\hline 16 & 38 & M & 303 & $\mathrm{~N}$ & 2004 & $\mathrm{EFV}+\mathrm{FTC} / \mathrm{TDF}$ & $3 \mathrm{TC}$ & 74 & 24.3 & $\mathrm{~N}$ & $\mathrm{~N}$ & 969 & $<50$ & $\mathrm{~N}$ & 3.4 & 0.3 & 0.80 \\
\hline 17 & 37 & M & 163 & $\mathrm{~N}$ & 2005 & $\begin{array}{l}\text { ATV/r+ } \\
\text { 3TC/ABC }\end{array}$ & $\mathrm{LPV} / \mathrm{r}$ & 68 & NK & $\mathrm{N}$ & $\mathrm{N}$ & 362 & $<50$ & $\mathrm{~N}$ & 4.3 & 0.6 & 1.21 \\
\hline 18 & 37 & M & 9 & $\mathrm{~N}$ & 2004 & $\mathrm{ATV}+3 \mathrm{TC} / \mathrm{ABC}$ & $\begin{array}{l}\text { LPV/r, AZT, ATV/r, TDF, } \\
\text { FTC }\end{array}$ & 65 & NK & $\mathrm{Y}$ & $\mathrm{N}$ & 349 & $<50$ & $\mathrm{~N}$ & 5.3 & 0.3 & 0.62 \\
\hline 19 & 32 & M & 326 & $\mathrm{~N}$ & 2004 & AZT/3TC/ABC & TDF, EFV & 79 & 25.3 & $\mathrm{~N}$ & $\mathrm{~N}$ & 424 & $<50$ & $\mathrm{Y}$ & 5.3 & 0.7 & 1.01 \\
\hline 20 & 34 & M & 399 & $\mathrm{~N}$ & 2006 & EFV+ FTC/TDF & $\mathrm{LPV} / \mathrm{r}$ & 74 & 29 & Y & $\mathrm{N}$ & 624 & $<50$ & Y & 4.6 & 0.4 & 0.65 \\
\hline 21 & 53 & M & 23 & Y & 2004 & ATV/r+ FTC/TDF & $\mathrm{LPV} / \mathrm{r}, 3 \mathrm{TC}$ & 63 & NK & $\mathrm{Y}$ & $\mathrm{N}$ & 325 & $<50$ & $\mathrm{Y}$ & 5.4 & 0.3 & 0.80 \\
\hline 22 & 40 & M & 497 & $\mathrm{~N}$ & 2005 & ATV/r+ FTC/TDF & LPV/r, EFV & 63 & 18 & $\mathrm{~N}$ & $\mathrm{~N}$ & 1139 & $<50$ & $\mathrm{~N}$ & 3.6 & 0.2 & 0.84 \\
\hline 23 & 53 & M & 122 & $\mathrm{~N}$ & 1997 & $\mathrm{ATV} / \mathrm{r}+\mathrm{FTC} / \mathrm{TDF}$ & $\begin{array}{l}\text { AZT, 3TC, d4T, ddI, ddC, } \\
\text { ABC, IDV, NFV, EFV, NVP }\end{array}$ & 53 & 31 & $\mathrm{~N}$ & $\mathrm{~N}$ & 351 & $<50$ & $\mathrm{Y}$ & 4.6 & 0.3 & 0.91 \\
\hline 24 & 66 & M & 424 & $\mathrm{~N}$ & 1999 & NVP+ FTC/TDF & AZT, ABC, ATV/r & 78 & 29 & $\mathrm{~N}$ & $\mathrm{~N}$ & 690 & $<50$ & Y & 6 & 0.6 & 1.90 \\
\hline
\end{tabular}

F: female; M: male; N: no; Y: yes; NK: not known; NVP: nevirapine; FTC: emtricitabine; TDF: tenofovir; AZT: zidovudine; 3TC: lamivudine; ABC: abacavir; ATV/r: atazanavir/ritonavir; FSV/r: fosamprenavir/ritonavir; LPV/r: lopinavir/ritonavir; EFV: efavirenz; ddI: didanosine; d4T: stavudine; ddC: zalcitabine; IDV: indinavir; NFV: nelfinavir; KPa: KiloPascal; COL: total serum cholesterol; TG: serum triglycerides.

affect about $30-40 \%$ of them [17-20]. The risk of NAFLD is increased in patients with a body mass index (BMI) > $25 \mathrm{Kg} / \mathrm{m}^{2}$ and a greater BMI together with a waist circumference $>102 \mathrm{~cm}$ for men are associated with severity and progression of the disease [20]. In our cohort, BMI was $>25 \mathrm{Kg} / \mathrm{m}^{2}$ in all 13 patients with liver steatosis and 
available BMI, in $3 / 3$ with progressive fibrosis at elastography, and in 3/4 patients with mild fibrosis at least in one biochemical score of fibrosis (BMI was not available for $1 / 4$ patients). Therefore, our results confirm that overweight is associated with liver steatosis [21]. At the same time, transient elastography and fibrosis markers could be useful to pick-up patients with more severe degree of liver damage, who can benefit from more aggressive interventions (diet, exercise and, probably, metformin).

The study patients were all on HAART and most of them experienced several different drugs in their past treatment. Thus, hypertransaminasemia could likely be an effect of drug toxicity. However, our results suggest that certain antiretroviral drugs are not the only culprit. Among the three patients with fibrosis at elastometry, one (patient \#13) did not experience either nevirapine (a drug that has been associated with frequent transaminase elevations [22] and with liver fibrosis according to one study [23], but not to another [24]), or didanosine (a drug whose risk of mithocondrial toxicity is well known, possibly leading to liver steatosis and fibrosis [25]). Moreover, the length of antiretroviral drug exposure was very limited in this patient (11 months overall) and 2/5 patients with mild fibrosis detected with biochemical non-invasive markers did not experience any of the most dangerous drugs.

Therefore, in absence of any major causes of liver fibrosis, the potential impact of other factors merit attention. Among these factors, HIV by itself has been implicated: interestingly, significant mitochondrial alterations were found in the liver of patients who had never used antiretroviral medications [26].

The present study presents some limitations. First, the sample was small and the study was cross-sectional. In our small population of HIV positive patients with transaminase elevations, no subjects presented with cirrhosis or noncirrhotic portal hypertension. This finding is reassuring but may simply reflect the fact that this phenomenon are either rare or did not occurred yet in this small group of patients. Large, perspective studies are therefore mandatory. Indeed, non-cirrhotic portal hypertension has been described as possibly associated with HIV infection itself or with antiretroviral therapy [27]. Moreover, portal hypertension in HIV positive patients was correlated with didanosine exposure and removal of this drugs preceded clinical and laboratory improvements [28].

Second, the patients did not undergo liver biopsy, the gold standard for the diagnosis of liver fibrosis and more sophisticated analyses to assess mitochondrial toxicity were not performed (e.g., electronic microscopy or biochemical analysis of the respiratory chain).

Third, the fact that there is a discordance between the non invasive methods for detecting fibrosis could be considered a limitation of the study, if our aim is to evaluate the prevalence of fibrosis in the study cohort, since liver biopsy was not performed and the diagnosis was only obtained through non invasive methods. Moreover, our results underline the need of larger and more powerful studies to describe the prevalence of fibrosis in HIV not-coinfected patients. At the same time, further studies are needed to check the concordance between non invasive markers of fibrosis in monoinfected HIV patients.

In conclusion, our study confirms that liver steatosis is a frequent problem in HIV-positive patients, even after excluding alcohol abuse, co-infections and other causes of liver disease. By contrast, liver fibrosis was rare in our populations and not inivariably linked to the use of "fibrogenic" antiretroviral drugs. Importantly, striking discordance between non invasive methods to detect liver fibrosis or cirrhosis was found, indicating that further studies are necessary to improve diagnostic algorithms (with or without liver biopsy). In the meantime, ultrasonography, ultrasonic transient elastography and biochemical fibrosis scores may help select patients with more severe liver disease, who can benefit from more aggressive diagnosis (e.g. liver biopsy) and treatment interventions.

\section{REFERENCES}

[1] Soriano V, Puoti M, Garcia-Gasco P, et al. Antiretroviral drugs and liver injury. AIDS 2008; 22: 1-13.

[2] Sulkowski M, Mehta S, Torbenson M, et al. Hepatic steatosis and antiretroviral drug use among adults coinfected with HIV and hepatitis $C$ virus. AIDS 2005; 19: 585-592.

[3] Mc Govern B, Ditelberg J, Taylor L, Gandhi R, Christopoulos K, Chapman S. Hepatic steatosis is associated with fibrosis, nucleoside analogue use, and hepatitis $C$ virus genotype 3 infection in HIV-seropositive patients. Clin Infect Dis 2006; 43: 365-372.

[4] Bravo AA, Sheth SG, Chopra S. Liver biopsy. N Engl J Med 2001; 344: 495-500.

[5] Bedossa P, Dargere D, Paradis V. Sampling variability of liver fibrosis in chronic hepatitis C. Hepatology 2003; 38: 1449-1457.

[6] Yoneda M, Yoneda M, Mawatari H, et al. Noninvasive assessment of liver fibrosis by measurement of stiffness in patients with nonalcoholic fatty liver disease (NAFLD). Dig Liver Dis 2008; 40: 371-78.

[7] Cozzi Lepri A, Prosperi M, Lo Caputo S, et al. Fib4 is an independent predictor of serious liver disease among HIV-infected patients with or without $\mathrm{HBV} / \mathrm{HCV}$ co-infection in the Icona foundation study. Italian Conference on AIDS and Retroviruses Brescia, Italy. 20 -22 June 2010. Infection, 2010; 38 (Suppl 1): 73.

[8] DallaPiazza M, Amorosa VK, Localio R, Kostman JR, Lo Re V $3^{\text {rd }}$. Prevalence and risk factor for significant liver fibrosis among HIVmonoinfected patients. BMC Infect Dis. 2010 May 13; 10: 116.

[9] Blackard J, Welge J, Taylor L, et al. HIV mono-infection is associated with a non-invasive index of liver fibrosis in women: 2009: $17^{\text {th }}$ Conference on Retroviruses and Opportunistic Infections; 2009 Feb 16-19; San Francisco, USA [abstract 681].

[10] Wai CT, Greenson JK, Fontana RJ, et al. A simple non invasive index can predict both significant fibrosis and cirrhosis in patients with chronic hepatitis C. Hepatology 2003; 38: 518-526.

[11] Sterling RK, Lissen E, Clumeck N, et al. Development of a simple noninvasive index to predict significant fibrosis inpatients with HIV/HCV coinfection. Hepatology 2006; 43: 1317-1325.

[12] Shaheen AA, Myers RP. Diagnostic accuracy of the aspartate aminotransferase-to-platet ratio index for the prediction of hepatitis C-related fibrosis: a systematic review. Hepatology 2007; 46(3): 912-21.

[13] Vallet-Pichard A, Mallet V, Nalpas B, et al. FIB-4: an inexpensive and accurate marker of fibrosis in $\mathrm{HCV}$ infection. Comparison with liver biopsy and fibrotest. Hepatology 2007; 32: 32-36.

[14] Leroy V. Other non-invasive markers of liver fibrosis. Gastroenterol Clin Biol 2008; 32 (6 Suppl 1): 52-7.

[15] González Guilabert MI, Hinojosa Mena-Bernal C, del Pozo González J, del Pozo Pérez MA. Retrospective study of FibroScan, APRI, FIB-4 and FORNS indexes compared with liver biopsy in the evaluation of liver fibrosis in patients with chronic hepatitis $\mathrm{C}$ monoinfection and HIV coinfection. Gastroenterol Hepatol 2010; 33(6): 425-32.

[16] Angulo P. Nonalcoholic fatty liver disease. N Engl J Med 2002; 346: 1221-1231.

[17] Mohammed SS, Aghdassi E, Salit IE, et al. HIV-positive patients with non-alcoholic fatty liver disease have a lower body mass index 
and are more physically active than HIV-negative patients. J Acquir Immune Defic Syndr 2007; 45: 432-8.

[18] Moreno-Torres A, Domingo P, Pujol J, Blanco-Vaca F, Arroyo JA, Sambeat MA. Liver trygliceride content in HIV-infected patients on combination antiretroviral therapy studied with $1 \mathrm{H}-\mathrm{MR}$ spectroscopy. Antivir Ther 2007; 12: 195-203.

[19] Sutinen J, Häkkinen AM, Westerbacka J, et al. Increased fat accumulation in the liver in HIV-infected patients with antiretroviral therapy-associated lipodystrophy. AIDS 2002; 16: 2183-93.

[20] Guaraldi G, Squillace N, Stentarelli C, et al. Nonalcoholic fatty liver disease in HIV-infected patient referred to a metabolic clinic: prevalence, characteristics, and predictors. CID 2008: 47; 250-257.

[21] Church TS, Kuk JL, Ross R, Priest EL, Biltoft E, Blair SN. Association of cardiorespiratory fitness, body mass index, and waist circumference with non-alcoholic fatty liver disease. Gastroenterology 2006; 130: 2023-2030.

[22] Torti C, Costarelli S, De Silvestri A, et al. Analysis of severe hepatic events associated with nevirapine-containing regimens. Drug Safety 2007; 30(12): 1161-1169.
[23] Macìas J, Castellano V, Merchante N, et al. Effect of antiretroviral drugs on liver fibrosis in HIV-infected patients with chronic hepatitis C: harmful impact of nevirapine. AIDS 2004; 19(6): 767 74.

[24] Berenguer J, Bellòn JM, Miralles P, et al. Association between exposure to nevirapine and reduced liver fibrosis progression in patients with HIV and hepatitis C coinfection. Clin Infect Dis 2008; 46(1): 137-43.

[25] Nunez M. Hepatotoxicity of antiretrovirals: incidence, mechanisms and management. J Hepatol 2006; 44(1 Suppl): S132-9.

[26] Mendes-Correa MC, Andrade Jr HF, Takakura CF, Seixas-Duarte MI. Hepatic ultrastructural mithocondrial changes prior to antiretroviral therapy in HIV-infected patients in Brazil. J Intern Ass Ph AIDS Care 2008; 7 (5): 252-58.

[27] Saifee S, Joelson D, Braude J, et al. Noncirrhotic portal hypertension in patients with human immunodeficiency virus-1 infection. Clin Gastroenter and Hepat 2008; 6: 1167-1169.

[28] Maida I, Garcia-Gasco P, Sotgiu G, Rios MJ, Vispo ME, MartinCarbonero L. Antiretroviral-associated portal hypertension: a new clinical condition? Prevalence, predictors and outcome. Antivir Ther 2008; 13(1): 103-107.

(C) Izzo et al.; Licensee Bentham Open.

This is an open access article licensed under the terms of the Creative Commons Attribution Non-Commercial License (http://creativecommons.org/licenses/ by-nc/3.0/) which permits unrestricted, non-commercial use, distribution and reproduction in any medium, provided the work is properly cited. 